\title{
A Platform Incorporating Trimeric Antigens into Self-Assembling Nanoparticles Reveals SARS-CoV-2-Spike Nanoparticles to Elicit Substantially Higher Neutralizing Responses than Spike Alone
}

Baoshan Zhang $^{1 *}$, Cara W. Chao ${ }^{1 *}$, Yaroslav Tsybovsky ${ }^{2}$, Olubukola M. Abiona ${ }^{1}$, Geoffrey B. Hutchinson $^{1}$, Juan I. Moliva ${ }^{1}$, Adam S. Olia ${ }^{1}$, Amarendra Pegu ${ }^{1}$, Emily Phung ${ }^{1,3}$, Guillaume Stewart-Jones ${ }^{1}$, Raffaello Verardi ${ }^{1}$, Lingshu Wang ${ }^{1}$, Shuishu Wang ${ }^{1}$, Anne Werner ${ }^{1}$, Eun Sung Yang $^{1}$, Christina Yap ${ }^{1}$, Tongqing Zhou ${ }^{1}$, John R. Mascola ${ }^{1}$, Nancy J. Sullivan ${ }^{1}$, Barney S.

Graham $^{1}$, Kizzmekia S. Corbett ${ }^{1}$ and Peter D. Kwong ${ }^{1 \mathrm{II}}$

${ }^{1}$ Vaccine Research Center, National Institute of Allergy and Infectious Diseases, National Institutes of Health, Bethesda, Maryland, USA.

2 Electron Microscopy Laboratory, Cancer Research Technology Program, Leidos Biomedical Research Inc., Frederick National Laboratory for Cancer Research, Frederick, Maryland, USA.

${ }^{3}$ Institute for Biomedical Sciences, George Washington University, Washington, DC, USA.

* These authors contributed equally to this work.

II Corresponding author (PDK) Email: pdkwong@nih.gov 


\section{Abstract}

24 Antigens displayed on self-assembling nanoparticles can stimulate strong immune responses and

25 have been playing an increasingly prominent role in structure-based vaccines. However, the

26 development of such immunogens is often complicated by inefficiencies in their production. To

27 alleviate this issue, we developed a plug-and-play platform using the spontaneous isopeptide-bond

28 formation of the SpyTag:SpyCatcher system to display trimeric antigens on self-assembling

29 nanoparticles, including the 60-subunit Aquifex aeolicus lumazine synthase (LuS) and the 24-

30 subunit Helicobacter pylori ferritin. LuS and ferritin coupled to SpyTag expressed well in a

31 mammalian expression system when an $N$-linked glycan was added to the nanoparticle surface.

32 The respiratory syncytial virus fusion (F) glycoprotein trimer - stabilized in the prefusion

33 conformation and fused with SpyCatcher - could be efficiently conjugated to LuS-SpyTag or

34 ferritin-SpyTag, enabling multivalent display of F trimers with prefusion antigenicity. Similarly,

35 F-glycoprotein trimers from human parainfluenza virus-type 3 and spike-glycoprotein trimers from

36 SARS-CoV-2 could be displayed on LuS nanoparticles with decent yield and antigenicity.

37 Notably, murine vaccination with the SARS-CoV-2 spike-LuS nanoparticles elicited $~ 25$-fold

38 higher neutralizing responses, weight-per-weight relative to spike alone. The versatile platform

39 described here thus allows for multivalent plug-and-play presentation on self-assembling

40 nanoparticles of trimeric viral antigens, with SARS-CoV-2 spike-LuS nanoparticles inducing

41 particularly potent neutralizing responses. 


\section{Introduction}

Self-assembling nanoparticles are playing an increasingly prevalent role in vaccine

44 development as vaccine vehicles and immunomodulators. The appeal of nanoparticle immunogens

45 lies in their inherent multivalent display of antigens, which is known to elicit robust B cell

46 responses (reviewed in ${ }^{1}$ ). There have been numerous efforts to genetically fuse viral immunogens

47 to nanoparticles within prokaryotic and eukaryotic systems, utilizing direct genetic fusion of

48 antigenic molecules with self-assembling nanoparticle monomers ${ }^{2-4}$, chemical conjugation ${ }^{5,6}$, and a

49 spontaneous intramolecular isopeptide bond formation with the SpyTag:SpyCatcher system ${ }^{7,8}$, and

50 some of these nanoparticles are now entering clinical trials ${ }^{2,9,10}$.

51 Another important factor to consider in viral immunogen design is glycosylation. Viral

52 pathogens are often heavily glycosylated, often as a means to evade the human immune system.

53 Moreover, many viral antigens require glycosylation to be stably expressed and correctly folded.

54 Although several studies have described plug-and-play nanoparticle systems ${ }^{11-16}$, many use

55 prokaryotic expression systems, which are not suitable to produce correctly glycosylated antigens.

56 Furthermore, $\mathrm{N}$-glycans can be manipulated in immunogen design to selectively occlude unwanted

57 epitopes as well as to improve the solubility and stability of immunogens ${ }^{17-19}$. Another factor to

58 consider is that metastable type 1 fusion machines are prevalent vaccine targets ${ }^{20}$.

59 Here we developed a modular self-assembling nanoparticle platform that allows for the

60 plug-and-play display of trimeric viral glycoproteins on nanoparticle surfaces, utilizing the

61 SpyTag:SpyCatcher system. We assessed this system with three prefusion (preF)-stabilized viral

62 trimeric glycoproteins: respiratory syncytial virus fusion (RSV F) glycoprotein ${ }^{21}$, human

63 parainfluenza virus type 3 fusion glycoprotein (PIV3 F) ${ }^{22}$, and SARS-CoV-2 spike

64 glycoprotein ${ }^{23,24}$. SpyTag-coupled nanoparticles could be expressed with sufficient yield of soluble

65 proteins from a mammalian expression system after the addition of nanoparticle surface glycans. 
66 The nanoparticle-formatted trimers exhibited improved antigenicity versus soluble trimers for

67 apical epitopes, and we explicitly tested the immunogenicity for the nanoparticle-formatted

68 trimeric antigen from SARS-CoV-2 in mice. Overall, protein antigens and nanoparticle scaffolds

69 could be produced independently before conjugation, thereby expediting the otherwise generally

70 cumbersome process of making and troubleshooting immunogens genetically fused to self-

71 assembling nanoparticle subunits. Such a modular nanoparticle assembly platform may thus be a

72 useful tool for plug-and-play screening of trimeric viral immunogens in a multivalent highly

73 immunogenic context, and we provide proof-of-principle for increased immunogenicity of a

74 nanoparticle-displayed SARS-CoV-2 spike.

\section{Results}

77 Expression of LuS- and ferritin-nanoparticle scaffolds with SpyTag requires the addition of

\section{an $N$-linked glycan}

To construct a reliable plug-and-play platform for nanoparticle presentation of antigens, we chose Aquifex aeolicus lumazine synthase $(\mathrm{LuS})^{25}$ and Helicobacter pylori ferritin ${ }^{26}$ as nanoparticle scaffolds with SpyTag:SpyCatcher conjugation system ${ }^{15}$ to display antigens on

82 nanoparticle surface. The SpyTag:SpyCatcher system is highly specific and stable with an

83 isopeptide bond and has been used for conjugation of antigens on nanoparticle surfaces ${ }^{7,27}$ (Fig.

84 1a). LuS and ferritin have served as scaffolds for nanoparticle immunogens in several clinical

85 studies: for LuS see https://www.clinicaltrials.gov/ct2/show/NCT03699241 ${ }^{28}$; for ferritin, see

86 https://www.clinicaltrials.gov/ct2/show/NCT03547245 $5^{10,29,30}$. The $\mathrm{N}$ terminus of both ferritin and

$87 \mathrm{LuS}$ are exposed to the nanoparticle surface and are thus accessible for SpyTag or SpyCatcher

88 attachment (Fig. 1b). The $\mathrm{C}$ terminus of $\mathrm{LuS}$ is also accessible on the nanoparticle surface and can

89 be used to display purification tags. We designed mammalian expression constructs expressing 
90 fusion proteins of SpyTag or SpyCatcher with LuS or ferritin. The constructs included both His-

91 and Strep-tags for purification purposes, along with a signal peptide for secretion of the expressed

92 proteins into supernatant medium (Fig. 1b).

93 Initial constructs yielded low levels of soluble proteins for the nanoparticle-SpyTag or

94 SpyCatcher fusion proteins. To improve protein solubility and expression, we added glycans to the

95 surface of the nanoparticles, designing a panel of LuS and ferritin constructs with SpyTag and

96 SpyCatcher (Table 1 and Supplementary Table S1). For LuS constructs, we added a glycosylation

97 site at position 71 (PDB 1HQK numbering). For ferritin constructs, two potential glycosylation

98 sites (96 and 148) were tested. The addition of $N$-linked glycosylation sites facilitated expression

99 of soluble nanoparticles in the cell culture supernatant. Three of the constructs produced

100 appreciable yields of well-assembled nanoparticles, LuS with N71 and SpyTag at N-terminus

101 (hereafter referred to as LuS-N71-SpyTag), ferritin with N96 and SpyTag, and ferritin S148

102 (glycan at N146) and SpyTag (Table 1). Of the two ferritin constructs, the ferritin with N96 and

103 SpyTag had a higher yield and was chosen for further studies (hereafter referred to as ferritin-N96-

104 SpyTag). Size exclusion chromatography (SEC) and electron microscopy (EM) analyses indicated

105 that LuS-N71-SpyTag formed a homogeneous nanoparticle population in solution (Fig. 1c,d). The

106 ferritin-N96-SpyTag sample comprised mainly intact nanoparticles with some minor unassembled

107 species (Fig. 1c,d). Negative-stain electron microscopy (EM) images indicated both nanoparticles

108 to be well-assembled with expected $\operatorname{sizes}^{25,26}$ (Fig. 1d). Two-dimensional class average revealed

109 more detailed structural features of the nanoparticles, which were consistent with previously

110 published structures of the two nanoparticles. These data indicated the ferritin and LuS

111 nanoparticles were compatible with the SpyTag and glycosylation site addition. These alterations

112 were well tolerated, allowing for robust nanoparticle assembly. To verify the glycosylation of

113 LuS- and ferritin-SpyTag nanoparticles, we performed PNGase F digestion and checked for glycan 
114 cleavage through SDS-PAGE (Fig. 1e). Both nanoparticles showed a band shift in the presence of

115 PNGase F, indicating the presence of $N$-liked glycan on the nanoparticles and its removal by the

116 amidase digestion. While the glycan cleavage in LuS-N71-SpyTag is distinct, it is less apparent in

117 ferritin-N96-SpyTag, likely due to incomplete glycosylation of ferritin-N96-SpyTag and multiple

118 bands of ferritin on SDS-PAGE. Ferritin has been observed to exhibit a single band on SDS-PAGE

119 in some studies ${ }^{2}$ but multiple bands in others ${ }^{16,31}$, presumably due to protease cleavage at the C

120 terminus or incomplete glycosylation. However, these different sized ferritin molecules assembled

121 correctly as nanoparticles with expected dimensions as indicated by SEC and EM (Fig. 1c,e).

122

123 Conjugation of RSV F-SpyCatcher to LuS-N71-SpyTag displays prefusion RSV F 'DS2'-

124 trimers homogeneously on the surface of the LuS-N71-Spylinked-RSV F nanoparticle

125 With no effective licensed vaccine against respiratory syncytial virus (RSV), the causative

126 agent for a prevalent childhood disease that results in approximately 60,000 hospitalizations and

12710,000 annual deaths in the US, developing an immunogen capable of eliciting protection is of

128 paramount importance ${ }^{32}$. Previous efforts in developing a vaccine capable of eliciting protective

129 antibodies have resulted in the identification of RSV trimers stabilized in its prefusion

130 conformation, RSV F DS-Cav1 (DS-Cav1), and RSV F DS2 (DS2) ${ }^{21,33}$. DS2 was shown to elicit

131 higher RSV neutralization responses than DS-Cav1 ${ }^{33}$. With this as our motivation and as a test

132 case for our nanoparticle SpyTag:SpyCatcher system, we investigated the feasibility of displaying

133 DS2 in the context of nanoparticle immunogens.

134 We prepared DS2 coupled to SpyCatcher (hereafter referred to as RSV F-SpyCatcher) by

135 genetic engineering to append SpyCatcher to the C-terminus of RSV F after a 3 residue (GSG)

136 linker (Supplementary Table S1). After expression and purification, we conjugated the purified

137 RSV F-SpyCatcher to the purified 60-mer LuS-N71-SpyTag nanoparticle (Fig. 2a). SEC profiles 
138 of the two components revealed that LuS-N71-SpyTag eluted around $13 \mathrm{~mL}$ and RSV F-

139 SpyCatcher eluted near $15 \mathrm{~mL}$ on Superdex 200 Increase 10/300 column (GE Health Sciences)

140 (Fig. 2b). The conjugated LuS-N71-SpyLinked-RSV F-SpyCatcher nanoparticle (LuS-N71-

141 SpyLinked-RSV F) eluted in a new peak at $\sim 10 \mathrm{~mL}$ by SEC (Fig. 2b). SDS-PAGE showed the

142 appearance of species of larger molecular weight of $\sim 90 \mathrm{kDa}$ in the conjugation mixture, followed

143 by bands of residual LuS-N71-SpyTag monomer and RSV F-SpyCatcher components at $20 \mathrm{kDa}$

144 and $60 \mathrm{kDa}$, respectively (Fig. 2c), confirming the success of the conjugation reaction. To estimate

145 the conjugation efficiency, we measured the intensity of each band on the SDS-PAGE gel image

146 of the conjugated nanoparticle product (Fig. 2c), as a surrogate of mass for each component.

147 Taking into consideration the molecular weight of each component, we calculated the molar ratio

148 of each component to total protein in the sample. We estimated $67 \%$ of all the LuS nanoparticle

149 subunit was conjugated to RSV F trimer. To verify particle integrity after conjugation, we

150 performed negative stain EM following SEC purification. LuS-N71 -SpyTag conjugated with RSV

151 F-SpyCatcher efficiently produced uniform particles with a core diameter of $18.2 \pm 1.0 \mathrm{~nm}$

152 decorated with trimer spike of $11.4 \pm 0.8 \mathrm{~nm}$ in length (Fig. 2d). We then confirmed the prefusion

153 state of the LuS-N71-SpyLinked RSV F nanoparticle through surface plasmon resonance using

154 RSV prefusion F specific antibodies D25 (site Ø) and MPE8 (site III) (Fig. 2e) ${ }^{21}$. Notably, RSV F

155 on nanoparticles showed an enhanced on-rate to the apex-targeting D25 antibody and reduced on-

156 rate to the equatorial targeting MPE8 versus trimeric RSV F, a crucial antigenic characteristic

157 signifying appropriate nanoparticle display ${ }^{3}$. 

out to conjugate the 24-mer ferritin-N96-SpyTag with RSV F-SpyCatcher in the same manner

163 (Fig. 3a). SEC of ferritin-N96-SpyTag nanoparticle showed a peak at around 16-17 mL, slightly

164 slower than RSV F-SpyCatcher (Fig. 3b). Negative stain EM revealed that ferritin-N96-SpyTag

165 formed nanoparticle of the expected size (Fig. 1c). The conjugation mixture of ferritin-N96-

166 SpyTag with RSV F-SpyCatcher exhibited a peak at $10 \mathrm{~mL}$ (void volume of the SEC column),

167 suggesting successful formation of the conjugation product (referred to as ferritin-RSV F) (Fig.

168 3b). SDS-PAGE demonstrated the appearance of a new band at $\sim 90 \mathrm{kDa}$, the expected size of

169 ferritin-N96-SpyLinked-RSV F nanoparticle, with residual ferritin-N96-SpyTag at around $20 \mathrm{kDa}$

170 (Fig. 3c). Using the same method as for LuS-N71-SpyLinked-RSV F above, we estimated 85\% of

171 all the ferritin nanoparticle subunit was conjugated to RSV F trimer. To confirm the formation of

172 ferritin-N96-SpyLinked-RSV F nanoparticle, we performed negative stain EM, which showed

173 well-formed nanoparticles with the expected size and shape, displaying trimer spikes around the

174 ferritin nanoparticle (Fig. 3d).

175 To verify the conserved prefusion state of the conjugated RSV F trimer, we measured the 176 binding of ferritin-N96-SpyLinked-RSV F to D25 and MPE8 IgGs through SPR (Fig. 3e).

177 Importantly, we observed the on-rate to increase for D25, which recognizes an epitope at the

178 trimer apex, but the on-rate to decrease for MPE8, which recognizes an equatorial epitope on the

179 trimer, similar to the observation for LuS-N71-SpyLinked-RSV F.

181 Displaying PIV3 F glycoprotein trimer on LuS nanoparticle via SpyTag:SpyCatcher

182 conjugation improves antibody binding to the trimer apex

183 To demonstrate the plug-and-play versatility of the SpyTag:SpyCatcher nanoparticle

184 system, we produced PIV3 F glycoprotein trimer ${ }^{34}$ as a fusion protein with SpyCatcher at the C 
185 terminus and conjugated with the LuS-N71-SpyTag nanoparticle (Fig. 4, a-c, Supplementary Table

186 S1), similar to that described above for the conjugation of RSV F-SpyCatcher. PIV3 is a prevalent

187 human parainfluenza virus that causes respiratory illnesses, especially in infants and young

188 children $^{35,36}$. The conjugation mixture of PIV3 F-SpyCatcher and LuS-N71-SpyTag was loaded

189 onto the SEC column to purify the conjugated nanoparticle product LuS-N71-SpyLinked-PIV3 F

190 from unconjugated nanoparticles and the PIV3 F SpyCatcher trimer (Fig. 4b). SDS-PAGE analysis

191 revealed that the conjugated product had the expected molecular weight and there was not

192 unconjugated PIV3 F-SpyCatcher in the conjugation mixture (Fig. 4c). Using the same method as

193 for LuS-N71-SpyLinked-RSV F in the previous section, we estimated $68 \%$ of all the LuS

194 nanoparticle subunit was conjugated to PIV3 F trimer. The PIV3 F conjugated nanoparticle was

195 further verified through negative stain EM, which showed well defined trimer spikes decorating

196 the LuS nanoparticle with the expected size (Fig. 4d).

197 Having produced nanoparticles of LuS-N71-SpyTag conjugated with PIV3 F-SpyCatcher, 198 we next evaluated binding of LuS-N71-SpyLinked-PIV3 F with antibodies PIA174 and PIA75 ${ }^{34}$,

199 using SPR (Fig. 4e). The head-targeting antibody PIA174 showed an improved binding to LuS-

200 N71-SpyLinked-PIV3 F relative to its binding to PIV3 F-SpyCatcher. The stem-targeting antibody

201 PIA75, however, showed decreased binding to LuS-N71-SpyLinked-PIV3 F compared with PIV3

202 F-SpyCatcher.

204 Conjugation of SARS-CoV-2 spike trimer to LuS nanoparticle via SpyTag:SpyCatcher

205 displays the spike trimers homogeneously on the nanoparticle surface

Severe acute respiratory syndrome coronavirus 2 (SARS-CoV-2) caused the COVID-19

207 pandemic that is ongoing worldwide ${ }^{37}$. An effective vaccine against SARS-CoV-2 and related

208 coronaviruses is urgently needed. The SARS-CoV-2 spike glycoprotein trimer mediates virus-cell 
209 membrane fusion and thus a target for vaccine development ${ }^{23,24}$. To test the versatility of our plug-

210 and-play SpyTag:SpyCatcher nanoparticle system, we expressed and purified SARS-CoV-2 spike

211 fused with a C-terminal SpyCatcher and conjugated to the LuS-N71-SpyTag nanoparticle (Fig. 5,

212 a-c, Supplementary Table S1). For this construct, we used the prefusion stabilized version of spike

213 developed by McLellan and colleagues ${ }^{24}$, which included GSAS and PP mutations and the T4

214 phage fibritin trimerization domain along with a single-chain $\mathrm{Fc}$ tag as described by Zhou and

215 colleagues $^{38}$.

216 The conjugation mixture was loaded onto an SEC column to purify the conjugated

217 nanoparticle product LuS-N71-SpyLinked-CoV spike from unconjugated LuS-N71-SpyTag and

218 SARS-CoV-2 spike-SpyCatcher (Fig. 5b). SDS-PAGE analysis revealed the conjugated product to

219 have the expected molecular weight, and unconjugated spike-SpyCatcher was not observed after

220 conjugation (Fig. 5c). Using the same method as for LuS-N71-SpyLinked-RSV F, we estimated

$22191 \%$ of all the LuS nanoparticle subunit was conjugated to the spike trimer. Negative stain EM

222 showed LuS-N71-SpyLinked-CoV-2 spike nanoparticle to exhibit the expected size with spike

223 trimers displaying on the LuS nanoparticle surface (Fig. 5d). SPR measurements showed LuS-

224 N71-SpyLinked-SARS-CoV-2 Spike to bind to CR3022 $2^{39,40}$, an antibody targeting the receptor-

225 binding domain (RBD), indicating successful nanoparticle presentation of the spike trimer using

226 the LuS-SpyTag:SpyCatcher system.

228 LuS-N71-SpyLinked-nanoparticle display increases potential of SARS-CoV-2 spike to elicit

229 neutralizing antibodies

230 To assess immunogenicity, we injected mice with the LuS-N71-SpyLinked-CoV-2 spike

231 nanoparticle or spike trimers (stabilized by 2P mutation) $)^{24,41}$, or mock (LuS-N71-SpyTag)

232 nanoparticles at weeks 0 and 3 (Fig. 6a). Serum samples were collected two weeks after each 
233 immunization. After the first immunization, at the lowest immunogen dose of $0.08 \mu \mathrm{g}$, spike

234 nanoparticle-immune sera exhibited an anti-SARS-CoV-2 spike ELISA geometric mean titer of

2355,116 , whereas only 1 out of 10 trimeric spike-immunized sera exhibited a measurable titer (Fig.

236 6b); after a second immunization, titers for the spike nanoparticle-immune sera increased

237 substantially, by approximately 25 -fold. Immunizations with higher doses of spike nanoparticle

$238(0.4$ and $2.0 \mu \mathrm{g})$ increased titers more incrementally, both at week 2 and at week 5 . By contrast,

239 increases in dose of the spike trimer raised ELISA titers more substantially, with two of the mice

240 in the $2.0 \mu \mathrm{g}$ spike-trimer immune sera reaching the assay upper limit of detection with a titer of

$241 \quad$ 1,638,400 (Fig. 6b).

242 Importantly, pseudovirus neutralization assays revealed the LuS-N71-SpyLinked-CoV-2

243 spike nanoparticle to elicit potent neutralization responses with geometric mean $\mathrm{ID}_{50}$ titers of 413 ,

2441820 , and 1501 for immunization doses of $0.08,0.4$, and $2 \mu \mathrm{g}$, respectively (Fig. 6c). In

245 comparison, two doses of trimeric spike elicited neutralization titers at the 0.4 and $2 \mu \mathrm{g}$ doses with

246 a geometric mean $\mathrm{ID}_{50}$ of 49 and 315 , respectively, with no measurable neutralization at the 0.08

$247 \mu \mathrm{g}$ dose. In essence, $0.08 \mu \mathrm{g}$ of spike nanoparticle elicited a neutralization response that was

248 higher, though statistically indistinguishable from $2 \mu \mathrm{g}$ of trimeric spike. This indicated $\sim 25$-fold

249 higher immunogenicity on a weight-by-weight basis for the spike nanoparticle versus spike alone,

250 suggesting a substantial "dose-sparing" effect. Overall, presentation of the SARS-CoV-2 spike on

251 the LuS nanoparticle surface significantly improved its immunogenicity and required a lower

252 immunogen dose to elicit potent neutralization responses compared with the trimeric form.

\section{Discussion}

255 Nanoparticle-based immunogens can induce potent neutralizing antibodies ${ }^{2,3,42}$ and thus

256 may be promising vaccine candidates. To develop nanoparticle vaccine immunogens, rapid and 
257 efficient methods would help produce nanoparticle scaffolds that can be mixed and matched with

258 different immunogens. Previous efforts utilizing the spontaneous isopeptide bond formation with

259 the SpyTag:SpyCatcher system for nanoparticle surface display of immunogens ${ }^{11-16}$ have proven

260 the versatility of this system for antigen display. However, none of these previously published

261 reports utilized mammalian expression allowing for post-translational modifications, such as $N$ -

262 linked glycosylation. Here, we describe two nanoparticle platforms, lumazine synthase and ferritin,

263 for the display of trimeric viral protein immunogens using the SpyTag:SpyCatcher system. By

264 adding $N$-linked glycosylation sites to nanoparticle monomers, we were able to produce SpyTag-

265 coupled nanoparticles using mammalian cell culture.

LuS and ferritin nanoparticle platforms vary in the number of molecules displayed on the surface. LuS-N71-SpyTag contains 60 SpyTags whereas ferritin-N96-SpyTag has 24 displayed on surface, available for SpyCatcher-carrying molecules to couple to. Both platforms showed efficient conjugation of trimeric immunogens and formed nanoparticle rapidly under physiological conditions for RSV F, PIV3 F and SARS-CoV-2 spike trimers. One advantage of the LuS nanoparticle is the high efficiency of its particle assembly. The glycosylated LuS-N71-SpyTag

272 assembled into a homogenous particle that exhibited a single peak in size exclusion 273 chromatography.

To demonstrate the versatility of our SpyTag-displaying nanoparticles in immunogen

275 development, we conjugated them to three viral antigens of vaccine interest, the DS2-preF

276 stabilized RSV F ${ }^{33}$, a DS2-stabilized version of PIV3 $\mathrm{F}^{34}$, and the 2P-stabilized version of SARS-

277 CoV-2 spike ${ }^{24}$. In each of these, we appended the SpyCatcher after the 'foldon' heterologous

278 trimeric stabilization motif ${ }^{43}$. Conjugation of SpyTag-nanoparticles with SpyCatcher-coupled RSV

279 F, PIV3 F and SARS-CoV-2 spike trimers resulted in proper particle assembly. In all three cases,

280 we observed high conjugation efficiency. 
Our antigenicity analyses indicate that presentation of trimeric antigens from viral

282 pathogens on self-assembling nanoparticles needs to take into consideration the accessibility of the

283 antigenic epitopes. When a trimer protein is conjugated to a nanoparticle, such as LuS or ferritin in

284 this study, the trimer molecules are densely displayed on the nanoparticle surface. As a result of

285 the dense display, the epitopes near the nanoparticle surface, such as those at the stem region of the

286 trimers in this study, are less accessible to antibodies than the epitopes on the apex of trimer

287 molecules. Consequently, we observed an increased level of antibody binding to epitopes on the

288 apex and a decreased level of antibody binding to epitopes on the equatorial or stem region of

289 RSV F and PIV3 F trimer molecules (Figs. 2e, 3e and 4e).

290 The increased antigenicity of the apical epitopes on the trimer conjugated to nanoparticles

291 is expected to yield increased immunogenicity - especially at lower dose, and we provide proof-

292 of-principle for this with murine immunization studies with LuS-N71-SpyLinked-CoV-2 spike as

293 compared to soluble spike. As observed in prior studies ${ }^{2,3}$, nanoparticle immunogens elicited

294 stronger immune responses than the corresponding trimers at low immunogen doses: at the 0.08

$295 \mu \mathrm{g}$ dose after two immunizations, spike nanoparticle elicited neutralization response with ID $_{50}$ of

296 413, whereas trimeric spike elicited an equivalent neutralization titer only at the 25-fold higher

297 dose of $2 \mu \mathrm{g}$. At $0.4 \mu \mathrm{g}$, spike nanoparticle elicited $\sim 37$-fold higher $\mathrm{ID}_{50}$ than trimeric spike.

298 However, at a high dose of $2 \mu \mathrm{g}$, spike nanoparticle-elicited neutralization response appeared to

299 plateau - at a level $\sim 5$-fold higher in neutralization titer than the trimeric immunogen. Similar

300 increases in immunogenicity and with dose-sparing have been recently reported for nanoparticles

301 incorporating the receptor-binding domain (RBD) of the spike ${ }^{44}$. Overall, multivalent presentation

302 of trimeric antigens on nanoparticle can significantly improve their immunogenicity, allowing for

303 elicitation of potent immune responses at a relatively low immunogen dose. Our 
304 SpyTag:SpyCatcher system provides a versatile platform for preparation of such nanoparticle

305 immunogens from trimeric antigens.

306 It will be interesting to see if the plug-and-display technology described here will allow

307 for the incorporation of different molecules on multiple nanoparticles. Such molecules could

308 include not only trimeric viral immunogens, but immunostimulatory components, or molecules

309 targeting antigen presenting cells. Thus, the LuS- and ferritin-SpyTag displaying nanoparticles

310 described here may be amendable to mix-and-match display of immunogens and of

311 immunostimulatory or targeting components.

312

\section{Materials and Methods}

\section{Protein production and purification}

315 The amino acid sequences of protein expression constructs are listed in Supplementary Table S1.

316 For protein expression, $3 \mathrm{ml}$ of Turbo293 transfection reagent (Speed BioSystems) was mixed

317 with $50 \mathrm{ml}$ Opti-MEM medium (Life Technology) and incubated at room temperature (RT) for 5

318 min. $1 \mathrm{mg}$ plasmid DNAs was mixed with $50 \mathrm{ml}$ of Opti-MEM medium in a separate tube, and the

319 mixture added to the Turbo293 Opti-MEM mixture. The transfection mixture was incubated for 15

$320 \mathrm{~min}$ at RT then added to $800 \mathrm{ml}$ of Expi293 cells (Life Technology) at 2.5 million cells/ml. The

321 transfected cells were incubated overnight in a shaker incubator at $9 \% \mathrm{CO}_{2}, 37{ }^{\circ} \mathrm{C}$, and $120 \mathrm{rpm}$.

322 On the second day, about $100 \mathrm{ml}$ of Expi293 expression medium was added. On day 5 post

323 transfection, supernatants were harvested, filtered. Proteins were purified from the supernatant

324 using Ni-NTA and strep chromatography. SARS-CoV-2 spike-SpyCatcher was expressed as a

325 fusion protein with a single-chain Fc purification tag and purified using Protein A

326 chromatography. SARS-CoV-2 spike-SpyCatcher protein was cleaved off from Protein A column 
327 by HRV3C protease. All proteins were further purified by size exclusion chromatography on

328 Superdex 200 Increase 10/300 GL in PBS.

\section{LuS- and ferritin- SpyTag conjugations}

331 A 1:1 molar ratio of LuS- or ferritin-SpyTag and immunogen-SpyCatcher components were

332 combined and incubated at ambient temperature for approximately 3 hours, followed by size

333 exclusion column on Superdex200 Increase 10/300 GL in PBS to separate conjugated products

334 from residual components. The conjugated nanoparticle product was then run through SDS-PAGE

335 to verify conjugation and analyzed by negative-stain EM.

337 Negative-stain electron microscopy (EM)

338 Samples were diluted to $0.02-0.05 \mathrm{mg} / \mathrm{ml}$ with a buffer containing $10 \mathrm{mM}$ HEPES, $\mathrm{pH}$ 7, and 150

$339 \mathrm{mM} \mathrm{NaCl}$. A 4.7- $\mu 1$ drop of the diluted sample was applied to a glow-discharged carbon-coated

340 copper grid for approximately $15 \mathrm{~s}$. The drop was removed using blotting paper, and the grid was

341 washed three times with $4.7-\mu 1$ drops of the same buffer. Adsorbed proteins were negatively

342 stained by applying consecutively three $4.7-\mu 1$ drops of $0.75 \%$ uranyl formate and removing each

343 drop with filer paper. Micrographs were collected using SerialEM ${ }^{45}$ on an FEI Tecnai T20 electron

344 microscope operated at $200 \mathrm{kV}$ and equipped with an Eagle CCD camera or using EPU on a

345 ThermoFisher Talos F200C electron microscope operated at $200 \mathrm{kV}$ and equipped with a Ceta

346 CCD camera. The pixel size was 0.44 and $0.25 \mathrm{~nm}$ for Tecnai T20 and Talos F200C, respectively.

347 Particles were picked automatically using in-house written software (Y.T., unpublished).

348 Reference-free 2D classification was performed with Relion $1.4^{46}$ and SPIDER ${ }^{47}$. The dimensions

349 of VLP cores and spikes were measured with e2display.py program from EMAN2.1 software

350 package $^{48}$ using a representative micrograph (LuS-N71-SpyLinked-CoV-2 S) or 2D class average 
351 images (all other VLPs). For CoV-2 nanoparticles we observed increased structural content at low

$352 \mathrm{pH}$, whereas the other micrographs were collected at physiological $\mathrm{pH}$.

\section{Surface plasmon resonance (SPR)}

355 To prepare the chips (GE Healthcare Life Sciences CM5 chips), antibody IgG were immobilized

356 onto the chip by amine coupling to $\sim 100-1000$ response units (RU) depending the level of binding

357 of each trimer and nanoparticle pair to antibodies. To measure binding of SpyCatcher proteins and

358 and nanoparticles, a dilution series of SpyCatcher-linked proteins and nanoparticles were flowed

359 through the IgG sensor chip for $200 \mathrm{~s}$ followed by $800 \mathrm{~s}$ of dissociation at a flow rate of 30

$360 \mu \mathrm{L} / \mathrm{min}$. The starting trimer concentration for each sample was $200 \mathrm{nM}$. Sensor chip surfaces were

361 regenerated after each injection following manufacture instructions with Glycine 2.5 (GE

362 Healthcare Life Sciences $10 \mathrm{mM}$ glycine $-\mathrm{HCl}$ at $\mathrm{pH} 2.5$ ) at a flow rate of $40 \mu \mathrm{L} / \mathrm{min}$ for $180 \mathrm{~s}$.

\section{Mouse immunization}

365 Mouse experiments were carried out in compliance with National Institutes of Health regulations

366 and approval from the Animal Care and Use Committee of the Vaccine Research Center. Six week

367 old female BALB/cJ mice (Jackson Laboratories) were inoculated intramuscularly with Sigma

368 Adjuvant System, at weeks 0 and 3, as detailed previously ${ }^{49}$. Serum was collected 2 weeks post-

369 prime and post-boost for measurements of antibody responses as detailed hereafter.

\section{Enzyme-linked immunosorbent assay (ELISA)}

372 ELISA experiments were carried out as previously described ${ }^{41}$. Briefly, Nunc Maxisorp ELISA

373 plates (ThermoFisher) were coated with $100 \mathrm{ng} /$ well of stabilized soluble SARS-CoV-2 spike

$374 \operatorname{protein}^{24}$ (with His-tag cleaved to remove potential cross-reactivity) in $1 \mathrm{X} \mathrm{PBS}$ at $4{ }^{\circ} \mathrm{C}$ for $16 \mathrm{hr}$. 
375 To eliminate fold-on-specific binding, $50 \mu \mathrm{g} / \mathrm{mL}$ of fold-on protein was added to serial dilutions of

376 heat-inactivated sera for $1 \mathrm{hr}$ at room temperature (RT). After blocking in PBS-Tween (PBST)

377 supplemented with 5\% nonfat milk, plates were incubated with sera for $1 \mathrm{hr}$ at RT. After blocking

378 in PBS-Tween (PBST) supplemented with 5\% nonfat milk, plates were incubated with serial

379 dilutions of heat-inactivated sera for $1 \mathrm{hr}$ at RT. Secondary antibody, goat anti-mouse IgG

380 conjugated to horseradish peroxidase (ThermoFisher), was then added, followed by excitation with

$3813,5,3^{\prime} 5^{\prime}$-tetramethylbenzidine substrate (KPL). Each step in this procedure was followed by

382 standard washes in PBST. Endpoint titers were calculated as the dilution factor that resulted in an

383 optical density exceeding 4X background (secondary antibody alone).

\section{4}

385 Lentivirus-based pseudovirus neutralization assay

386 The pseudovirus neutralization assay was performed as described previously ${ }^{41,50}$. To produce

387 SARS-CoV-2 pseudovirus, a codon-optimized CMV/R-SARS-CoV-2 spike (Wuhan-1, Genbank

388 \#: MN908947.3) plasmid, was constructed and co-transfected with plasmids encoding luciferase

389 reporter, human transmembrane protease serine 2 (TMPRSS2) ${ }^{51}$, and lentivirus backbone into

390 HEK293T/17 cells (ATCC \#CRL-11268), as previously described ${ }^{52}$. Heat-inactivated serum was

391 mixed with the pseudovirus, incubated at $37^{\circ} \mathrm{C}$, and then added to ACE-2-expressing 293T cells.

392 Cells were lysed after $72 \mathrm{hr}$, and luciferase activity was measured. Percent neutralization was

393 calculated with uninfected cells as $100 \%$ neutralization and cells infected with only pseudovirus as

$3940 \%$ neutralization. $\mathrm{ID}_{50}$ titers were determined using a log (agonist) vs. normalized response

395 (variable slope) nonlinear function in Prism v8 (GraphPad).

397 Data availability

398 All relevant data are within the paper and its Supporting Information files. 


\section{References}

1 Lopez-Sagaseta, J., Malito, E., Rappuoli, R. \& Bottomley, M. J. Self-assembling protein nanoparticles in the design of vaccines. Comput Struct Biotechnol J 14, 58-68, doi:10.1016/j.csbj.2015.11.001 (2016).

2 Kanekiyo, M. et al. Self-assembling influenza nanoparticle vaccines elicit broadly neutralizing H1N1 antibodies. Nature 499, 102-106, doi:10.1038/nature12202 (2013).

3 Marcandalli, J. et al. Induction of Potent Neutralizing Antibody Responses by a Designed Protein Nanoparticle Vaccine for Respiratory Syncytial Virus. Cell 176, 1420-1431 e1417, doi:10.1016/j.cell.2019.01.046 (2019).

4 Swanson, K. A. et al. A respiratory syncytial virus (RSV) F protein nanoparticle vaccine focuses antibody responses to a conserved neutralization domain. Sci Immunol 5, doi:10.1126/sciimmunol.aba6466 (2020).

5 Cavelti-Weder, C. et al. Development of an Interleukin-1beta Vaccine in Patients with Type 2 Diabetes. Mol Ther 24, 1003-1012, doi:10.1038/mt.2015.227 (2016).

6 Cornuz, J. et al. A vaccine against nicotine for smoking cessation: a randomized controlled trial. PLoS One 3, e2547, doi:10.1371/journal.pone.0002547 (2008).

7 Zakeri, B. et al. Peptide tag forming a rapid covalent bond to a protein, through engineering a bacterial adhesin. Proc Natl Acad Sci U S A 109, E690-697, doi:10.1073/pnas.1115485109 (2012).

8 Marini, A. et al. A Universal Plug-and-Display Vaccine Carrier Based on HBsAg VLP to Maximize Effective Antibody Response. Front Immunol 10, 2931, doi:10.3389/fimmu.2019.02931 (2019).

9 He, L. et al. Presenting native-like trimeric HIV-1 antigens with self-assembling nanoparticles. Nat Commun 7, 12041, doi:10.1038/ncomms12041 (2016).

10 Yassine, H. M. et al. Hemagglutinin-stem nanoparticles generate heterosubtypic influenza protection. Nat Med 21, 1065-1070, doi:10.1038/nm.3927 (2015).

11 Escolano, A. et al. Immunization expands B cells specific to HIV-1 V3 glycan in mice and macaques. Nature 570, 468-473, doi:10.1038/s41586-019-1250-z (2019).

12 Govasli, M. L., Diaz, Y. \& Puntervoll, P. Virus-like particle-display of the enterotoxigenic Escherichia coli heat-stable toxoid STh-A14T elicits neutralizing antibodies in mice. Vaccine 37, 6405-6414, doi:10.1016/j.vaccine.2019.09.004 (2019).

13 Janitzek, C. M. et al. Bacterial superglue generates a full-length circumsporozoite protein virus-like particle vaccine capable of inducing high and durable antibody responses. Malar J 15, 545, doi:10.1186/s12936-016-1574-1 (2016).

14 Thrane, S. et al. Bacterial superglue enables easy development of efficient virus-like particle based vaccines. J Nanobiotechnology 14, 30, doi:10.1186/s12951-016-0181-1 (2016).

15 Brune, K. D. et al. Plug-and-Display: decoration of Virus-Like Particles via isopeptide bonds for modular immunization. Sci Rep 6, 19234, doi:10.1038/srep19234 (2016).

16 Wang, W. et al. Ferritin nanoparticle-based SpyTag/SpyCatcher-enabled click vaccine for tumor immunotherapy. Nanomedicine 16, 69-78, doi:10.1016/j.nano.2018.11.009 (2019).

17 Duan, H. et al. Glycan Masking Focuses Immune Responses to the HIV-1 CD4-Binding Site and Enhances Elicitation of VRC01-Class Precursor Antibodies. Immunity 49, 301-311 e305, doi:10.1016/j.immuni.2018.07.005 (2018).

18 Ingale, J. et al. Hyperglycosylated stable core immunogens designed to present the CD4 binding site are preferentially recognized by broadly neutralizing antibodies. J Virol $\mathbf{8 8 ,}$ 14002-14016, doi:10.1128/JVI.02614-14 (2014). 
19 Pantophlet, R., Wilson, I. A. \& Burton, D. R. Hyperglycosylated mutants of human immunodeficiency virus (HIV) type 1 monomeric gp120 as novel antigens for HIV vaccine design. J Virol 77, 5889-5901 (2003).

20 Rey, F. A. \& Lok, S. M. Common Features of Enveloped Viruses and Implications for Immunogen Design for Next-Generation Vaccines. Cell 172, 1319-1334, doi:10.1016/j.cell.2018.02.054 (2018).

21 McLellan, J. S. et al. Structure-based design of a fusion glycoprotein vaccine for respiratory syncytial virus. Science 342, 592-598, doi:10.1126/science.1243283 (2013).

22 Stewart-Jones, G. B. et al. Trimeric HIV-1-Env Structures Define Glycan Shields from Clades A, B, and G. Cell 165, 813-826, doi:10.1016/j.cell.2016.04.010 (2016).

23 Walls, A. C. et al. Structure, Function, and Antigenicity of the SARS-CoV-2 Spike Glycoprotein. Cell 181, 281-292 e286, doi:10.1016/j.cell.2020.02.058 (2020).

24 Wrapp, D. et al. Cryo-EM structure of the 2019-nCoV spike in the prefusion conformation. Science 367, 1260-1263, doi:10.1126/science.abb2507 (2020).

25 Zhang, X., Meining, W., Fischer, M., Bacher, A. \& Ladenstein, R. X-ray structure analysis and crystallographic refinement of lumazine synthase from the hyperthermophile Aquifex aeolicus at 1.6 A resolution: determinants of thermostability revealed from structural comparisons. J Mol Biol 306, 1099-1114, doi:10.1006/jmbi.2000.4435 (2001).

26 Cho, K. J. et al. The crystal structure of ferritin from Helicobacter pylori reveals unusual conformational changes for iron uptake. J Mol Biol 390, 83-98, doi:10.1016/j.jmb.2009.04.078 (2009).

27 Reddington, S. C. \& Howarth, M. Secrets of a covalent interaction for biomaterials and biotechnology: SpyTag and SpyCatcher. Curr Opin Chem Biol 29, 94-99, doi:10.1016/j.cbpa.2015.10.002 (2015).

28 Jardine, J. G. et al. HIV-1 broadly neutralizing antibody precursor B cells revealed by germline-targeting immunogen. Science 351, 1458-1463, doi:10.1126/science.aad9195 (2016).

29 Corbett, K. S. et al. Design of Nanoparticulate Group 2 Influenza Virus Hemagglutinin Stem Antigens That Activate Unmutated Ancestor B Cell Receptors of Broadly Neutralizing Antibody Lineages. mBio 10, doi:10.1128/mBio.02810-18 (2019).

30 Darricarrere, N. et al. Development of a Pan-H1 Influenza Vaccine. J Virol 92, doi:10.1128/JVI.01349-18 (2018).

31 Morris, C. D. et al. Differential Antibody Responses to Conserved HIV-1 Neutralizing Epitopes in the Context of Multivalent Scaffolds and Native-Like gp140 Trimers. mBio 8, doi:10.1128/mBio.00036-17 (2017).

32 Graham, B. S. Immunological goals for respiratory syncytial virus vaccine development. Curr Opin Immunol 59, 57-64, doi:10.1016/j.coi.2019.03.005 (2019).

33 Joyce, M. G. et al. Iterative structure-based improvement of a fusion-glycoprotein vaccine against RSV. Nat Struct Mol Biol 23, 811-820, doi:10.1038/nsmb.3267 (2016).

34 Stewart-Jones, G. B. E. et al. Structure-based design of a quadrivalent fusion glycoprotein vaccine for human parainfluenza virus types 1-4. Proc Natl Acad Sci U S A 115, 12265-12270, doi:10.1073/pnas.1811980115 (2018).

35 Jain, S. et al. Community-Acquired Pneumonia Requiring Hospitalization among U.S. Adults. N Engl J Med 373, 415-427, doi:10.1056/NEJMoa1500245 (2015).

36 Jain, S. et al. Community-acquired pneumonia requiring hospitalization among U.S. children. N Engl J Med 372, 835-845, doi:10.1056/NEJMoa1405870 (2015).

37 Cucinotta, D. \& Vanelli, M. WHO Declares COVID-19 a Pandemic. Acta Biomed 91, 157160, doi:10.23750/abm.v91i1.9397 (2020). 
38 Zhou, T. et al. Structure-Based Design with Tag-Based Purification and In-Process Biotinylation Enable Streamlined Development of SARS-CoV-2 Spike Molecular Probes. bioRxiv, 2020.06.22.166033, doi:10.1101/2020.06.22.166033 (2020).

39 ter Meulen, J. et al. Human monoclonal antibody combination against SARS coronavirus: synergy and coverage of escape mutants. PLoS Med 3, e237, doi:10.1371/journal.pmed.0030237 (2006).

40 Yuan, M. et al. A highly conserved cryptic epitope in the receptor-binding domains of SARSCoV-2 and SARS-CoV. Science, doi:10.1126/science.abb7269 (2020).

41 Corbett, K. S. et al. SARS-CoV-2 mRNA vaccine design enabled by prototype pathogen preparedness. Nature, doi:10.1038/s41586-020-2622-0 (2020).

42 Rts, S. C. T. P. Efficacy and safety of RTS,S/AS01 malaria vaccine with or without a booster dose in infants and children in Africa: final results of a phase 3, individually randomised, controlled trial. Lancet 386, 31-45, doi:10.1016/S0140-6736(15)60721-8 (2015).

43 Efimov, V. P. et al. Fibritin encoded by bacteriophage T4 gene wac has a parallel triplestranded alpha-helical coiled-coil structure. J Mol Biol 242, 470-486, doi:10.1006/jmbi.1994.1595 (1994).

44 Walls, A. C. et al. Elicitation of potent neutralizing antibody responses by designed protein nanoparticle vaccines for SARS-CoV-2. bioRxiv, 2020.08.11.247395, doi:10.1101/2020.08.11.247395 (2020).

45 Mastronarde, D. N. Automated electron microscope tomography using robust prediction of specimen movements. J Struct Biol 152, 36-51, doi:10.1016/j.jsb.2005.07.007 (2005).

46 Scheres, S. H. RELION: implementation of a Bayesian approach to cryo-EM structure determination. J Struct Biol 180, 519-530, doi:10.1016/j.jsb.2012.09.006 (2012).

47 Frank, J. et al. SPIDER and WEB: processing and visualization of images in 3D electron microscopy and related fields. J Struct Biol 116, 190-199, doi:10.1006/jsbi.1996.0030 (1996).

48 Tang, G. et al. EMAN2: an extensible image processing suite for electron microscopy. J Struct Biol 157, 38-46, doi:10.1016/j.jsb.2006.05.009 (2007).

49 Pallesen, J. et al. Immunogenicity and structures of a rationally designed prefusion MERSCoV spike antigen. Proc Natl Acad Sci U S A 114, E7348-E7357, doi:10.1073/pnas.1707304114 (2017).

50 Corbett, K. S. et al. Evaluation of the mRNA-1273 Vaccine against SARS-CoV-2 in Nonhuman Primates. New England Journal of Medicine, doi:10.1056/NEJMoa2024671 (2020).

51 Bottcher, E. et al. Proteolytic activation of influenza viruses by serine proteases TMPRSS2 and HAT from human airway epithelium. $J$ Virol 80, 9896-9898, doi:10.1128/JVI.01118-06 (2006).

52 Wang, L. et al. Evaluation of candidate vaccine approaches for MERS-CoV. Nature Communications 6, 7712, doi:10.1038/ncomms8712 (2015). 


\section{Acknowledgements}

532 We thank T. Beaumont and H. Spits for antibody D25, A. Lanzavecchia for antibodies PIA174,

533 PIA75 and MPE8, J. Stuckey for assistance with figures, and members of the Vaccine Research

534 Center for discussions or comments on the manuscript. We thank members of the NIH NIAID

535 VRC Translational Research Program for technical assistance with mouse experiments. Support

536 for this work was provided by the Intramural Research Program of the Vaccine Research Center,

537 National Institute of Allergy and Infectious Diseases, National Institutes of Health. This project

538 has been funded in part with Federal funds from Frederick National Laboratory for Cancer

539 Research, NIH, under Contract No. HHSN261200800001E (Y. Tsybovsky). K.S. Corbett is the

540 recipient of a research fellowship that was partially funded by the Undergraduate Scholarship

541 Program, Office of Intramural Training and Education, Office of the Director, NIH. The content of

542 this publication does not necessarily reflect the views or policies of the Department of Health and

543 Human Services, nor does mention of trade names, commercial products, or organizations imply

544 endorsement by the U.S. Government.

\section{Author contributions}

547 B.Z. and C.W.C. designed research with B.Z. heading protein design and production; B.Z.,

548 C.W.C., A.S.O., and R.V. produced nanoparticle and trimer proteins; C.W.C. and B.Z. prepared

549 trimer-coupled nanoparticles and performed antigenic assessments; Y.T. performed negative-stain

550 EM; S.W. assisted with manuscript assembly; T.Z. provided design for SARS-CoV-2 spike

551 protein; G.S-J. provided the design for PIV3 F protein; A.P., L.W. and E.S.Y. provided

552 pseudovirus; G.B.H. carried out immunizations; O.M.A. and A.W. performed ELISA; J.I.M.

553 performed neutralization assay; B.S.G. and K.S.C. designed mouse experiment and oversaw

554 ELISA experiment; J.R.M. oversaw pseudovirus preparation; N.J.S., B.S.G., and K.S.C. oversaw 
555 neutralization assay; E.P. and C.Y. performed data analyses for immunoassays; P.D.K. oversaw

556 the project with B.Z., C.W.C., S.W., and P.D.K. writing the paper, and all other authors providing

557 revisions and comments.

558

\section{Competing interests}

560 K.S.C. and B.S.G. are inventors on International Patent Application No. WO/2018/081318 entitled

561 "Prefusion Coronavirus Spike Proteins and Their Use.” K.S.C., O.M.A., G.B.H., and B.S.G. are

562 inventors on US Patent Application No. 62/972,886 entitled "2019-nCoV Vaccine”. 


\section{Figure legends}

564 Fig. 1. LuS- and ferritin-nanoparticle scaffolds with N-linked glycan and SpyTag express

\section{5 well as assembled nanoparticles in mammalian cells}

566 (a) Schematic diagram showing the separate SpyTag and SpyCatcher to combine through an

567 isopeptide bond as a means to covalently link molecules attached to SpyTag and molecules

568 attached to SpyCatcher. (b) Design of expression constructs to produce activated nanoparticles

569 with SpyTag in mammalian cells for conjugating antigens on the nanoparticle surface. Upper panel

570 shows the DNA construct. A SpyTag was placed at the N-terminus of the nanoparticle sequence

571 after the cleavable signal peptide. His and Strep tags were placed at the C-terminus of the LuS

572 nanoparticle. An N-linked glycosylation site was engineered in the nanoparticle sequence to

573 facilitate protein expression (see Table 1 and Supplementary Table S1 for more details). Lower

574 panels show the expected structures of the LuS-N71-SpyTag and ferritin-N96-SpyTag monomers

575 and assembled nanoparticles. Both glycan and SpyTag are expected to be on the nanoparticle

576 surface. (c) Size exclusion chromatograms confirmed the correct sizes of the nanoparticles. The

577 samples were loaded on a Superdex 200 Increase 10/300 GL column in PBS. Initial run of ferritin-

$57896 \mathrm{~N}-$ SpyTag nanoparticle revealed a tail of small molecular weight species; the chromatogram

579 shown here is the re-run main peak. (d) SDS-PAGE of LuS-N71-SpyTag and ferritin-N96-SpyTag

580 in the presence or absence of PNGase F. The position of PNGase F is marked. The multiple bands

581 for ferritin are likely due to proteolytic cleavage and incomplete glycosylation (see text). (e)

582 Negative stain EM images (left panels) and 2D class averages (right panels) of LuS-N71-SpyTag

583 and ferritin-N96-SpyTag show the correct assembly of the purified nanoparticles with expected

584 sizes. 
586 Fig. 2. Conjugation of RSV F-SpyCatcher to LuS-SpyTag displays prefusion RSV F trimer

587 homogenously on the surface of the LuS-N71-SpyLinked-RSV F nanoparticle

588 (a) Schematic diagram showing conjugation of SpyTag-coupled LuS to SpyCatcher-coupled RSV

589 prefusion F trimer to make LuS-N71-SpyLinked-RSV F nanoparticle. (b) SEC profiles of LuS-

590 N71-SpyTag, RSV F-SpyCatcher, and the conjugated product LuS-N71-SpyLinked-RSV F on a

591 Superdex 200 Increase 10/300 GL column in PBS. (c) SDS-PAGE of LuS-N71-SpyTag (lane 1),

592 RSV F-SpyCatcher (lane 2), and the conjugated LuS-N71-SpyLinked-RSV F nanoparticle product

593 (lane 3), in the presence of DTT. (d) Negative stain EM images of the LuS-N71-SpyLinked-RSV

594 F nanoparticle after SEC purification, showing (left panel) a representative micrograph and (right

595 panel) the 2D class averages. (e) Surface plasmon resonance of RSV F-SpyCatcher and LuS-N71-

596 SpyLinked-RSV F nanoparticle with prefusion-specific D25 IgG (site Ø) and MPE8 IgG (site III),

597 with IgG coupled to chip and nanoparticle in solution. A concentration series from $200 \mathrm{nM}$ to 1.56

$598 \mathrm{nM}$ of RSV F either as trimer (left) or couped to nanoparticle (right) was measured; $\mathrm{k}_{\mathrm{a}}$ values are

599 provided as these have been found to correlate with immunogenicity ${ }^{3}$.

600

601 Fig. 3. Conjugation of RSV prefusion F-SpyCatcher to ferritin-SpyTag produces uniform

602 ferritin-RSV F nanoparticles

603 (a) Schematic diagram showing the conjugation process of ferritin-N96-SpyTag and RSV F-

604 SpyCatcher to make ferritin-N96-SpyLinked-RSV F nanoparticle. (b) SEC profiles of ferritin-

605 N96-SpyTag, RSV F-SpyCatcher, and the conjugation reaction mixture on a Superdex 200

606 Increase 10/300 GL column in PBS. (c) SDS-PAGE of ferritin-N96-SpyTag (lane 1), RSV F-

607 SpyCatcher (lane 2), and the conjugated ferritin-N96-SpyLinked-RSV prefusion F SpyCatcher

608 nanoparticle product (lane 3), in the presence of DTT. Ferritin exhibited multiple bands due to

609 proteolytic cleavage and incomplete glycosylation (see text). (d) Negative stain EM images of the 
610 ferritin-RSV F nanoparticle after SEC purification, showing (left panel) a representative

611 micrograph and (right panel) the 2D class averages. (e) SPR of RSV F-SpyCatcher and Ferritin-

612 N96-SpyLinked-RSV F nanoparticle with prefusion-specific D25 IgG (site Ø) and MPE8 IgG (site

613 III) using immobilized IgG on sensor chip with nanoparticle and trimer in solution. A

614 concentration series from $200 \mathrm{nM}$ to $1.56 \mathrm{nM}$ of RSV F either as trimer (left) or coupled to

615 nanoparticle (right) was measured; $\mathrm{k}_{\mathrm{a}}$ values are provided.

616

617 Fig. 4. Conjugation of PIV3 F-SpyCatcher to LuS-SpyTag displays prefusion PIV3 F trimer

618 homogenously on the surface of the LuS-N71-SpyLinked-PIV3 F nanoparticle

619 (a) Schematic of the conjugation between LuS-N71-SpyTag and PIV3 F-SpyCatchert to produce

620 LuS-N71-SpyLinked-PIV3 F nanoparticle (b) SEC profiles of PIV3 F-SpyCatcher, LuS-SpyTag,

621 and the conjugated product LuS-N71-SpyLinked-PIV3 F on a Superdex 200 Increase 10/300 GL

622 in PBS. (c) SDS-PAGE of LuS-N71 (lane 1), PIV3 F-SpyCatcher (lane 2), and LuS-N71-

623 SpyLinked-PIV3 F conjugation mixture (lanes 3) in the presence of DTT. (d) Negative stain EM

624 of LuS-N71-SpyLinked-PIV3 following SEC showing a representative micrograph (left panel) and

625 2D class averages (right panel). (e) SPR measurements of PIV3 F-SpyCatcher and LuS-N71-

626 SpyLinked-PIV3 F were performed using IgG coupled chips with nanoparticle and timer in

627 solution. A concentration series from $200 \mathrm{nM}$ to $1.56 \mathrm{nM}$ of PIV3 F either as trimer (left) or

628 coupled to nanoparticle (right) was measured; $k_{a}$ values are provided.

630 Fig. 5. Conjugation of SARS-CoV-2 spike trimer to LuS-SpyTag displays SARS-CoV-2 spike

631 trimer on the surface of the LuS-N71-SpyLinked-CoV-2 spike nanoparticle

632 (a) Schematic diagram showing conjugation of SpyTag-coupled LuS to SpyCatcher-coupled

633 SARS-CoV-2 spike trimer to make LuS-N71-SpyLinked-CoV-2 spike nanoparticle. (b) SEC 
634 profiles of LuS-N71-SpyTag, SARS-CoV-2 spike-SpyCatcher, and the conjugated product LuS-

635 N71-SpyLinked-CoV-2 spike on a Superdex 200 Increase 10/300 GL column in PBS. (c) SDS-

636 PAGE of LuS-N71-SpyTag (lane 1), SARS-CoV-2 spike-SpyCatcher (lane 2), and the conjugation

637 mixture of LuS-N71-SpyTag with SARS-CoV-2 spike-SpyCatcher (lane 3) in the presence of

638 DTT. The conjugation mixture (lane 3) shows the conjugated LuS-N71-SpyLinked-CoV-2 spike

639 nanoparticle with minor excess of LuS-N71-SpyTag. (d) Negative stain EM of the LuS-N71-

640 SpyLinked-CoV-2 spike nanoparticle after SEC purification showing representative micrographs

641 (left panel) and 2D class average (right panel). (e) SPR response curves for LuS-N71-SpyLinked-

642 CoV-2 spike nanoparticle binding with RBD-targeting antibody CR3022 IgG, with IgG coupled to

643 chip and nanoparticle in solution. Because SARS-CoV-2 spike-SpyCatcher showed non-specific

644 binding only the coupled nanoparticle is shown. A series of nanoparticle concentrations was

645 analyzed in which the concentration of spike coupled to the nanoparticle ranged from $200 \mathrm{nM}$ to

$6461.56 \mathrm{nM}$. Observed $\mathrm{k}_{\mathrm{a}}$ value provided.

Fig. 6. Immunogenicity of LuS-N71-SpyLinked-CoV-2 spike

649 (a) Schematic immunization procedures for SARS-CoV-2 spike immunogens. (b) Serum

650 assessment of anti-SARS-CoV-2 spike ELISA titers. Immunization groups are color-coded.

651 Vertical dotted lines separate immunogen dose groups and weeks post prime. Starting reciprocal

652 serum dilution (100) is indicated with a horizontal dashed line. ELISA titer from each animal is

653 shown as an individual dot. Triangle-shape dot provided for ELISA titers at assay maximum.

654 Geometric means indicated by black horizontal lines. Note that the three animals immunized with

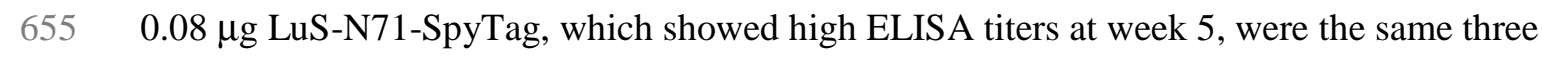

656 animals of this control group that showed detectable neutralization. (c) Neutralization titer from

657 each animal at week 5 is shown as an individual dot, and geometric means are indicated by black 
658 horizontal lines with values provided for each group. Immunization groups are color-coded as in

659 panel b. Limit of detection (titer $=40)$ indicated with a horizontal dashed line. $P$ values determined

660 by two-tailed Mann-Whitney tests. * indicates $P \leq 0.05$, ** indicates $P \leq 0.01$, *** indicates $P \leq$

$661 \quad 0.001$ and $* * * *$ indicates $P \leq 0.0001$.

662 
bioRxiv preprint doi: https://doi.org/10.1101/2020.06.11.147496: this version posted August 23, 2020. The copyright holder for this preprint (which was not certified by peer review) is the author/funder. This article is a US Government work. It is not subject to copyright under 17 USC 105 and is also made available for use under a CCO license.

663 Table 1. LuS- and ferritin-nanoparticles with SpyTag require the addition of $\boldsymbol{N}$-linked

664 glycans for expression.

\begin{tabular}{|c|c|c|c|c|}
\hline Construct ID & SpyTag & SpyCatcher & Position of glycan & Expression level $(\mathrm{mg} / \mathrm{L})$ \\
\hline \multicolumn{5}{|l|}{ Lumazine synthase } \\
\hline LuS-SpyTag no glycan & $\mathrm{x}$ & & None & $<0.1$ \\
\hline LuS-N71-SpyTag* & $\mathrm{x}$ & & 71 & 3.0 \\
\hline LuS-C-SpyCatcher no glycan & & $\mathrm{x}$ & None & $<0.1$ \\
\hline LuS-C71-SpyCatcher & & $\mathrm{x}$ & 71 & $<0.1$ \\
\hline LuS-N-SpyCatcher no glycan & & $\mathrm{x}$ & None & $<0.1$ \\
\hline LuS-N71-SpyCatcher & & $\mathrm{x}$ & 71 & $<0.1$ \\
\hline \multicolumn{5}{|l|}{ Ferritin } \\
\hline Ferritin-SpyTag no glycan & $\mathrm{x}$ & & None & $<0.1$ \\
\hline Ferritin-N96-SpyTag* & $\mathrm{x}$ & & 96 & 2.5 \\
\hline Ferritin-S148-SpyTag* & $\mathrm{x}$ & & 146 & 1.0 \\
\hline Ferritin-SpyCatcher no glycan & & $\mathrm{x}$ & None & $<0.1$ \\
\hline Ferritin-N96-SpyCatcher & & $\mathrm{x}$ & 96 & $<0.1$ \\
\hline Ferritin-S148-SpyCatcher & & $\mathrm{x}$ & 146 & $<0.1$ \\
\hline
\end{tabular}


bioRxiv preprint doi: https://doi.org/10.1101/2020.06.11.147496; this version posted August 23, 2020. The copyright holder for this preprint (which was not certified by peer review) is the author/funder. This article is a US Government work. It is not subject to copyright under 17 USC 105 and is also made available for use under a CCO license.

a

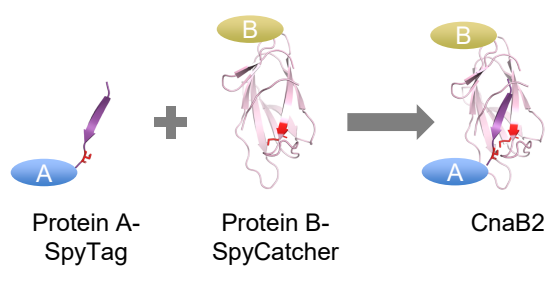

b Signal peptide SpyTag site (N71)

Nanoparticle-SpyTag mammalian cell expression construct

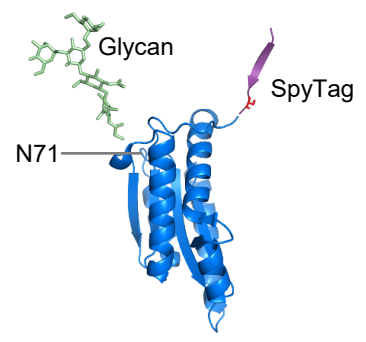

LuS-N71-SpyTag

(Monomer)

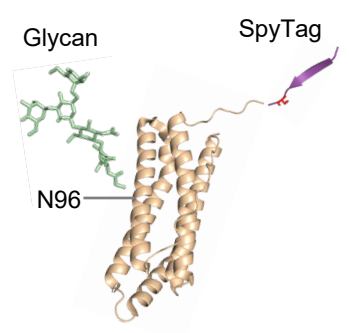

Ferritin-N96-SpyTag (Monomer)

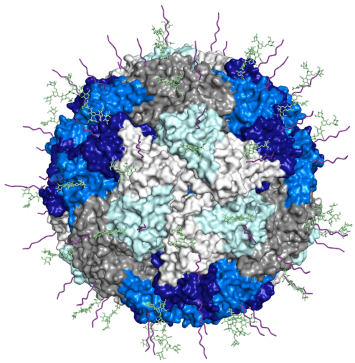

LuS-N71-SpyTag nanoparticle (60-mer)

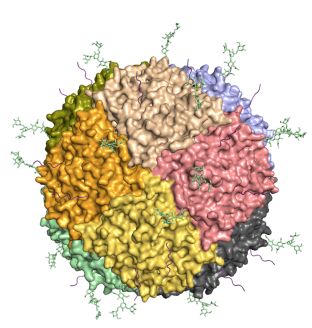

Ferritin-N96-SpyTag nanoparticle (24-mer)
C
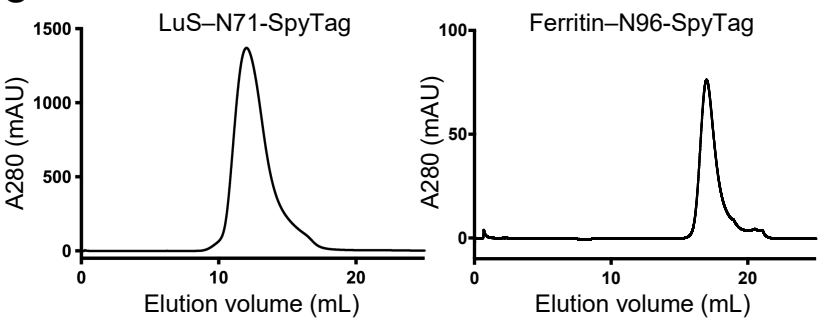

d

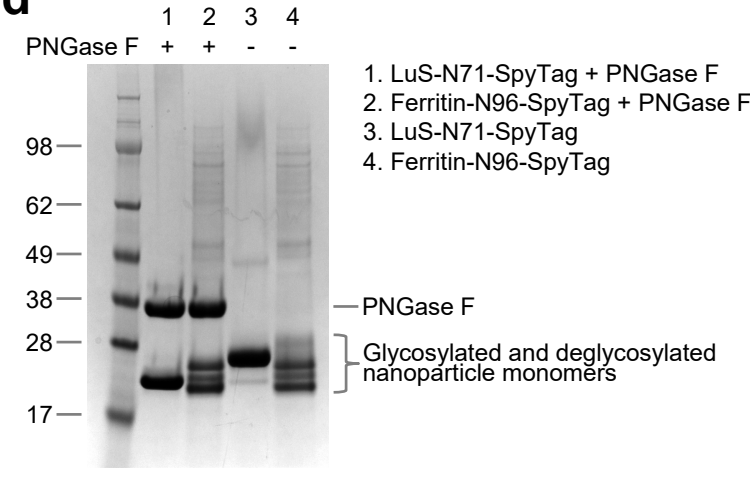

e

LuS-N71-SpyTag

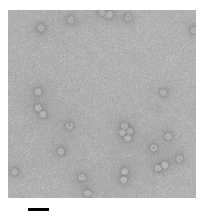

$50 \overline{\mathrm{nm}}$
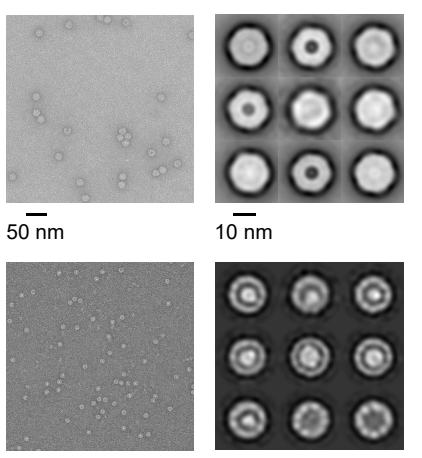

() 00
() 00
잉 0

$50 \overline{\mathrm{nm}}$ 
bioRxiv preprint doi: https://doi.org/10.1101/2020.06.11.147496; this version posted August 23, 2020. The copyright holder for this preprint (which was not certified by peer review) is the author/funder. This article is a US Government work. It is not subject to copyright under 17 USC 105 and is also made available for use under a CCO license.
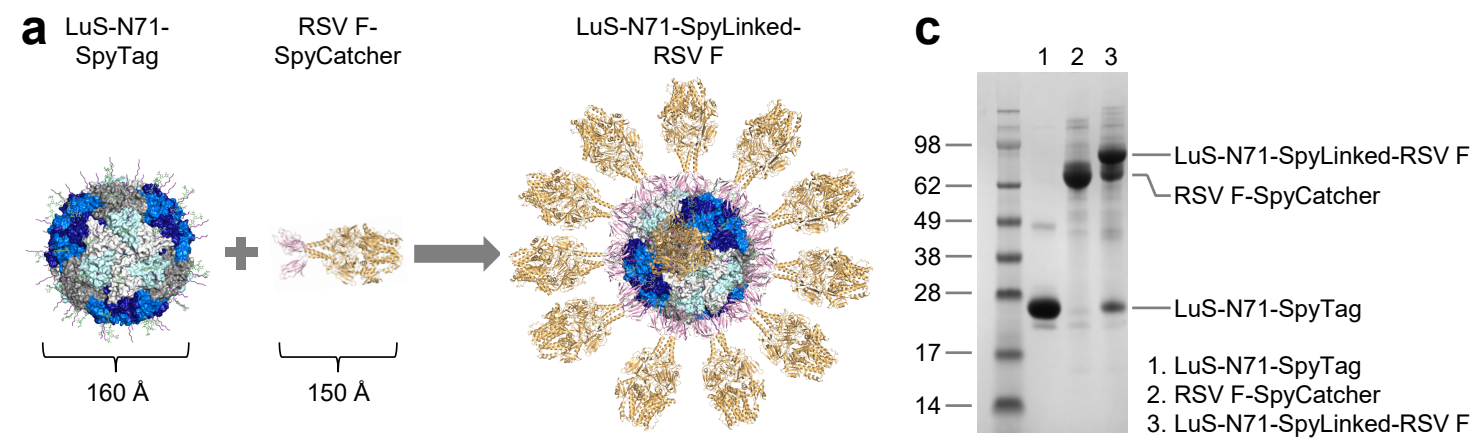

b
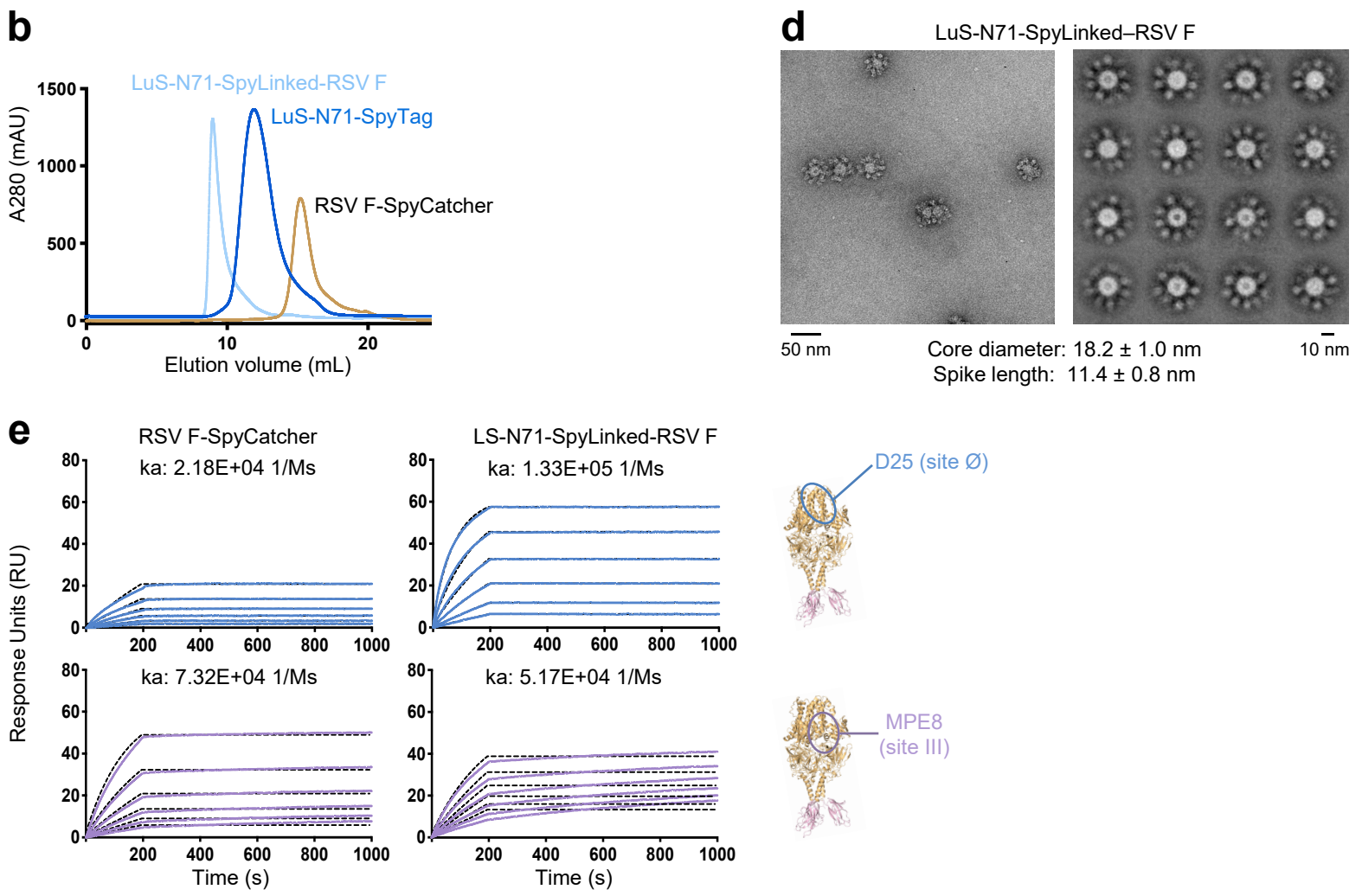
bioRxiv preprint doi: https://doi.org/10 1101/2020.06.11.147496: this version posted August 23, 2020. The copyright holder for this preprint (which was not certified by peer review) is the author/funder. This article is a US Government work. It is not subject to copyright under 17 USC 105 and is also made available for use under a CCO license.
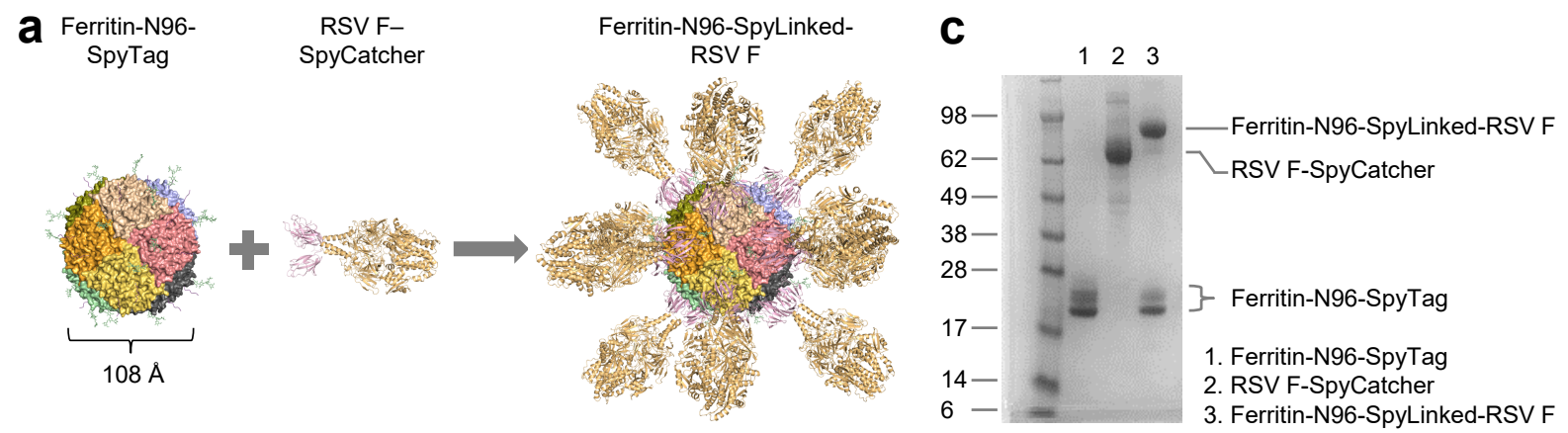

b
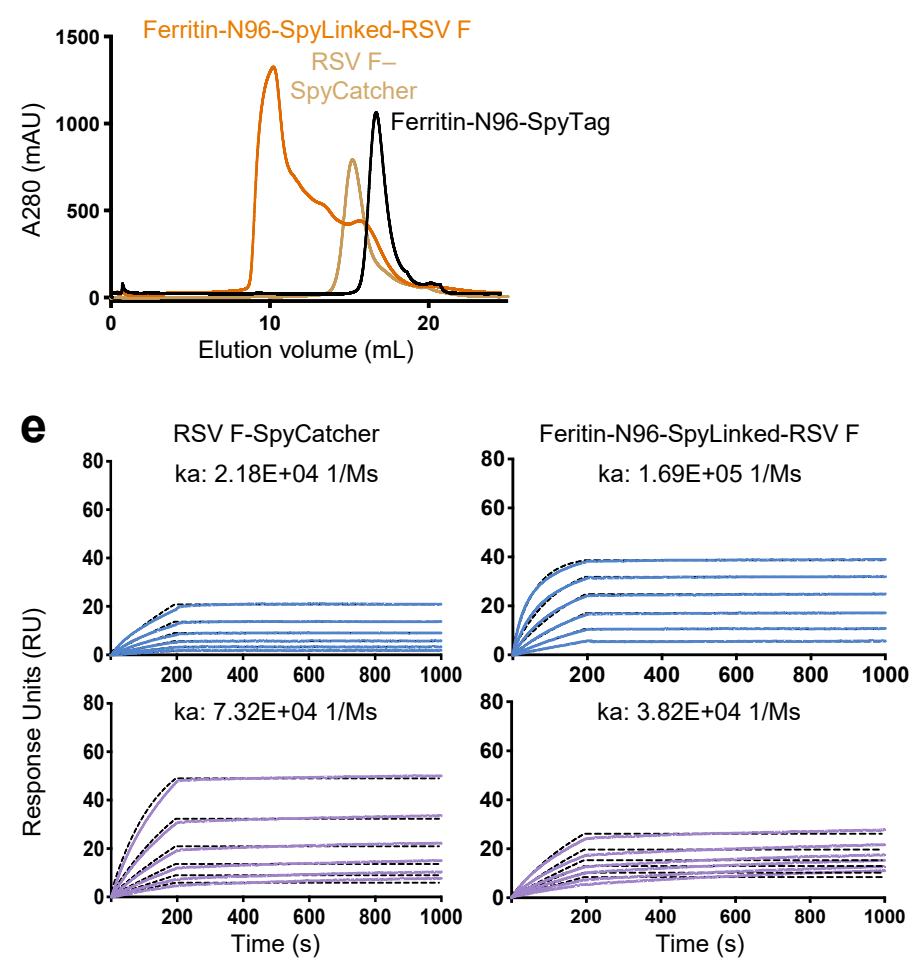

\section{d Ferritin-N96-SpyLinked-RSV F}
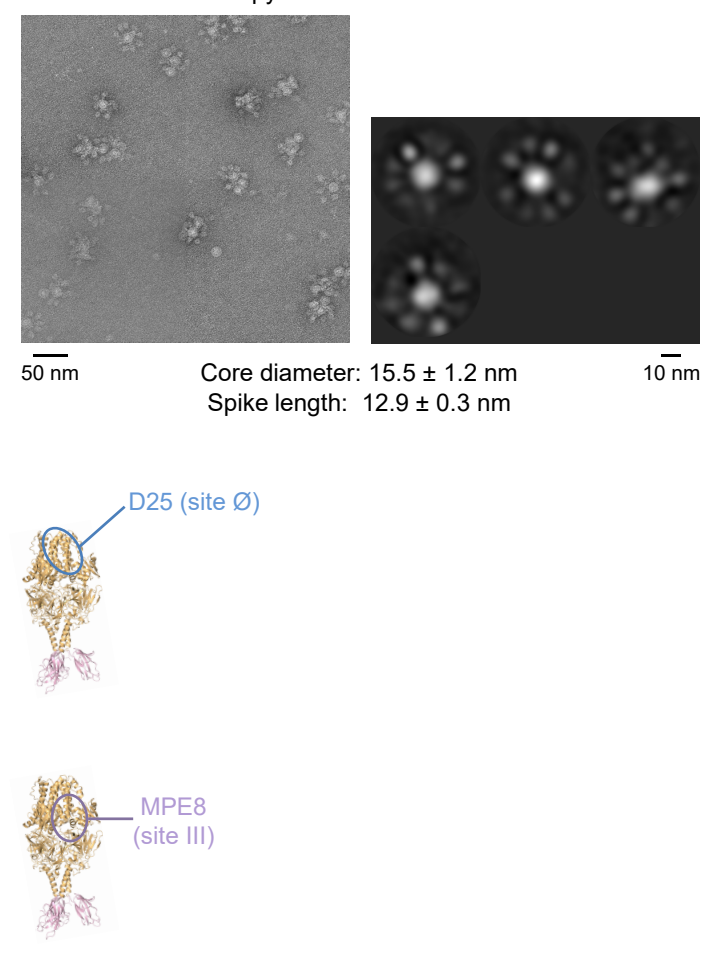
bioRxiv preprint doi: https://doi.org/10.1101/2020.06.11.147496; this version posted August 23, 2020. The copyright holder for this preprint (which was not certified by peer review) is the author/funder. This article is a US Government work. It is not subject to copyright under 17 USC 105 and is also made available for use under a CCO license.
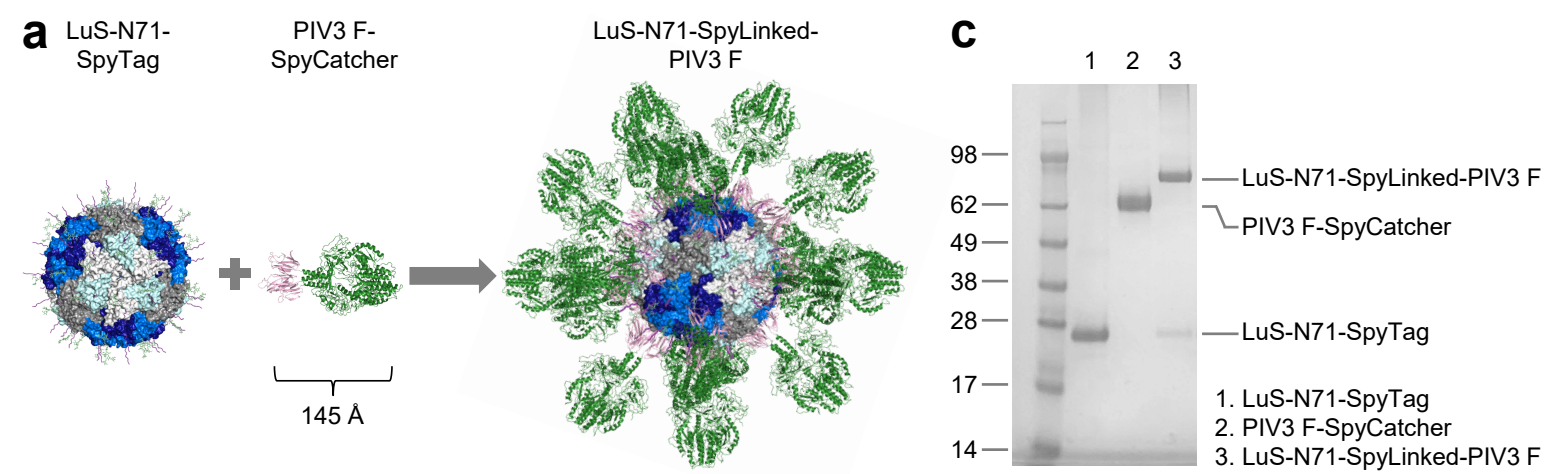

b

d LuS-N71-SpyLinked-PIV3 F
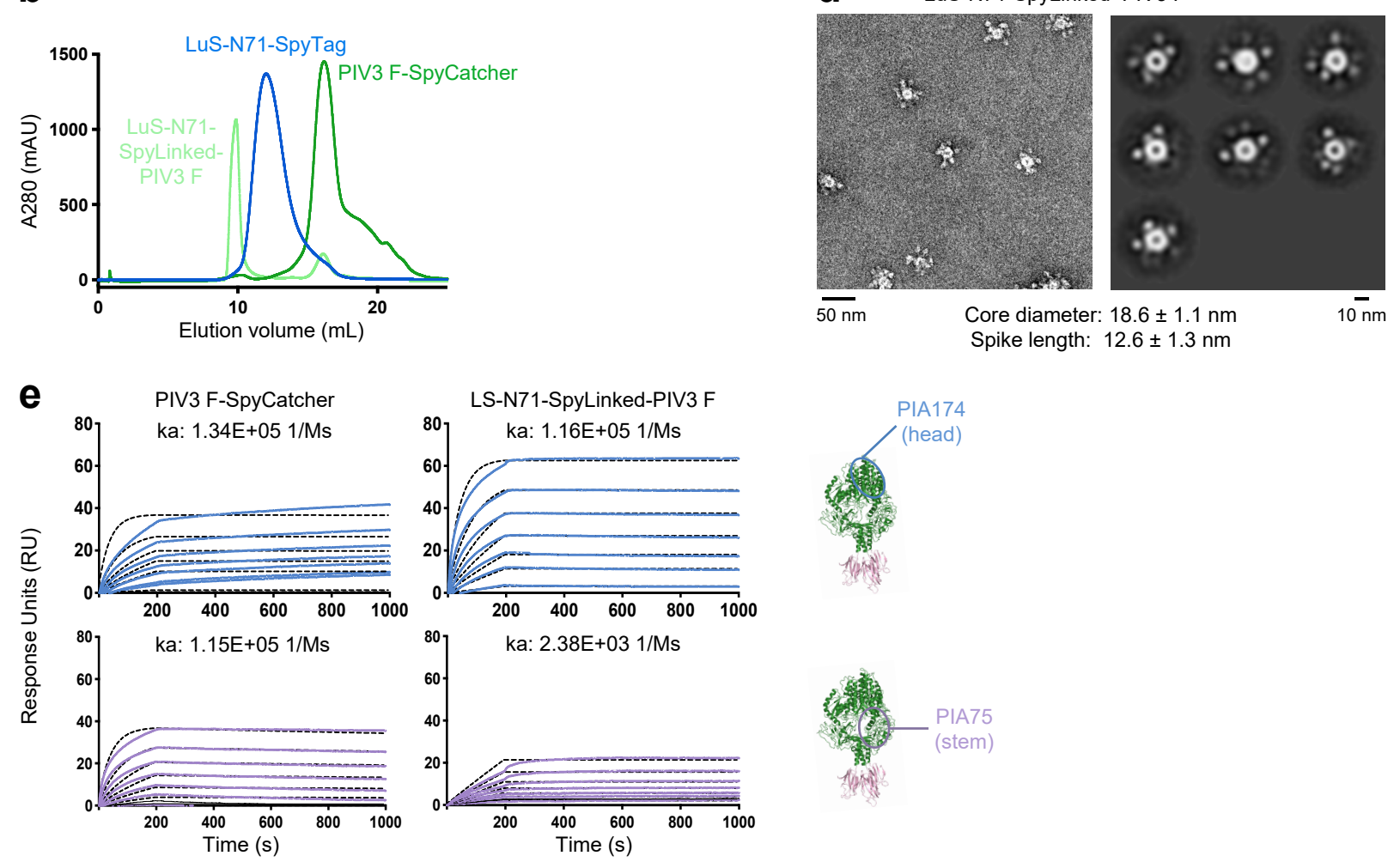
bioRxiv preprint doi: https://doi.org/10.1101/2020.06.11.147496; this version posted August 23, 2020. The copyright holder for this preprint (which was not certified by peer review) is the author/funder. This article is a US Government work. It is not subject to copyright under 17 USC 105 and is also made available for use under a CCO license.

$\begin{array}{cc}\text { a LuS-N71- } \\ \text { SpyTag } & \text { SARS-CoV-2 spike- } \\ \text { SpyCatcher }\end{array}$
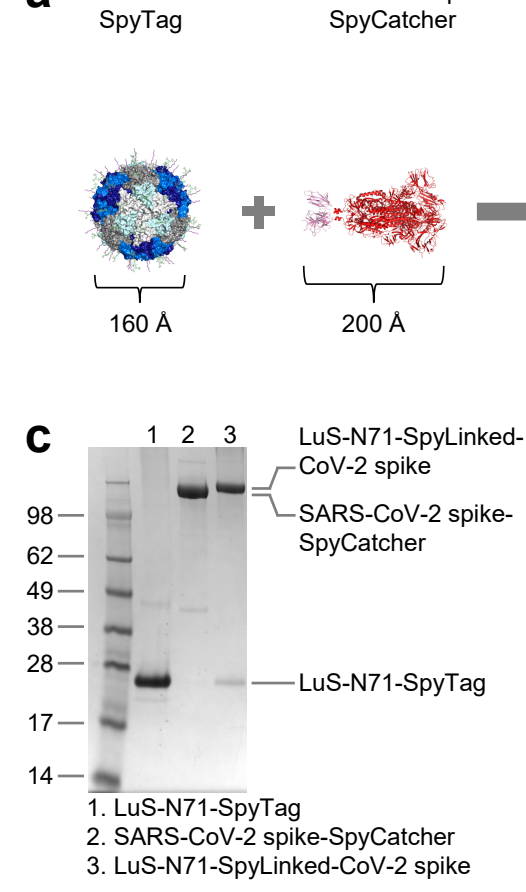

LuS-N71-SpyLinkedCoV-2 spike

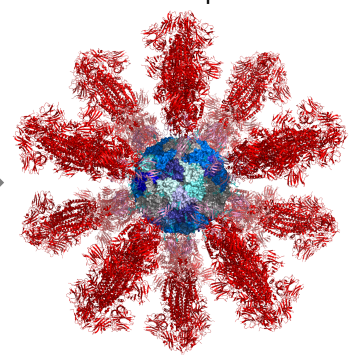

d

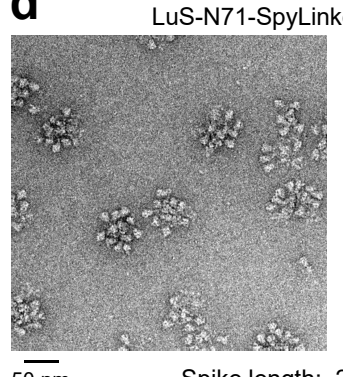

Spike length: $22.5 \pm 3.4 \mathrm{~nm}$ b
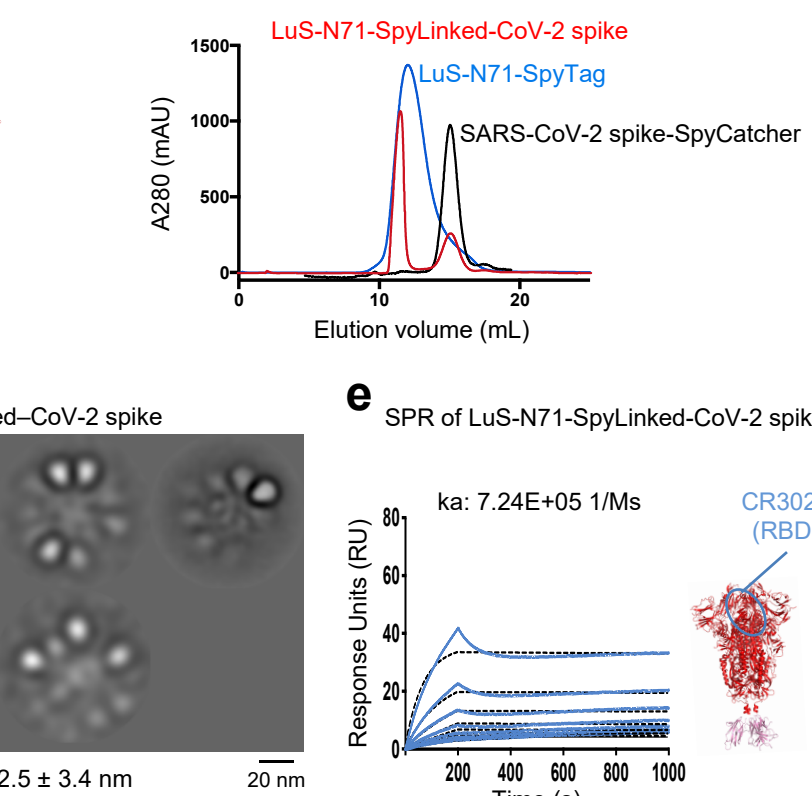

e SPR of LuS-N71-SpyLinked-CoV-2 spike

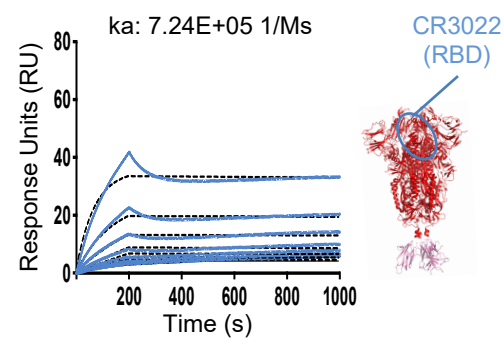


bioRxiv preprint doi: https://doi.org/10.1101/2020.06.11.147496: this version posted August 23, 2020. The copyright holder for this preprint (which was not certified by peer review) is the author/funder. This article is a US Government work. It is not subject to copyright under 17 USC 105 and is also made available for use under a CCO license.

a

Adjuvant: SAS

Route: i.m.

N=10/group

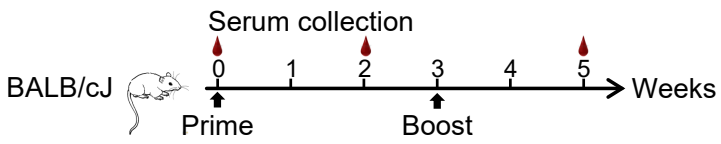

b

Anti-SARS-CoV-2 spike ELISA

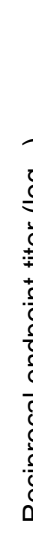

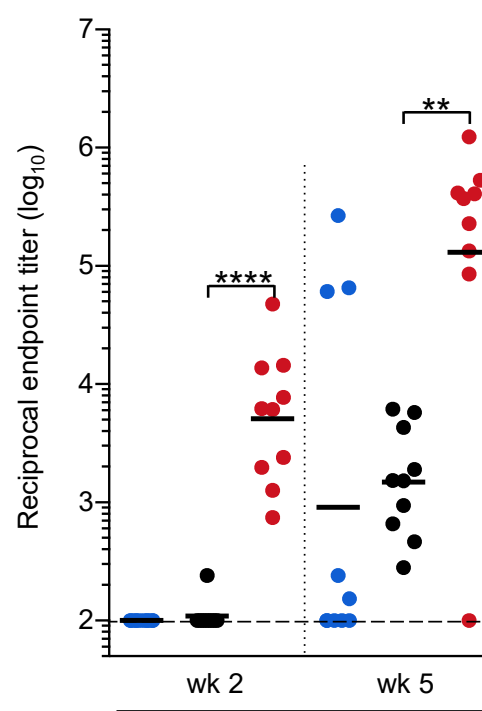

Dose $(\mu \mathrm{g})$

0.08
C 5 列 Serum neutralization titer at week 5

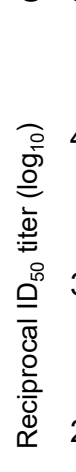
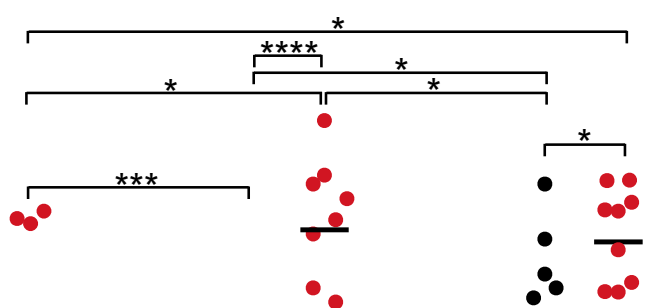

$\bar{\sigma}$

$\infty^{\bullet}$

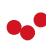

wk 2
- LuS-N71-SpyLinked-CoV-2 spike

- Spike ( $\Delta$ maximum)

- LuS-N71-SpyTag

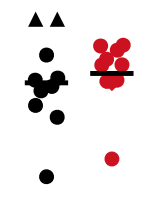

- LuS-N71-SpyLinked-CoV-2 spike

- Spike

- LuS-N71-SpyTag

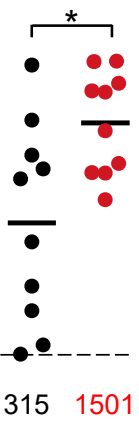

Geometric mean titers 